\title{
A generation of second harmonic of shear wave in elasto-plastic media
}

\author{
A.M. Doronin ${ }^{\dagger}$, V.I. Erofeev \\ †alexeydoronin@list.ru
}

Institute of Problems of Machine Science RAS, Belinsky St. 85, 603024, Nizhny Novgorod, Russia

\begin{abstract}
Elasto-plastic media, whose behavior is described by cross dependencies between first invariants of stress and strain tensors and second invariants of stress and strain deviators, is considered. The dependency has quadratic behavior in terms of shear strains. The goal of this article is to determine the possibility of generation of second harmonic of shear wave, whose appearance is not predicted by classical theory of elasticity, and the character of the process of energy transfer from first harmonic to second. In case of prevailing shear strains with only shear wave existing, the search of solutions of nonlinear equation, determining the dynamic behavior of the media, in form of travelling quasiharmonic waves with slowly changing amplitudes is performed. During the examination of the system of shortened equations for complex amplitudes, depending on time and travel distance, the unsymmetric character of harmonic interaction process is shown. It is determined that in stationary state even the presence of small plastic strains leads to appearance of doubled frequency (second harmonic) in the spectrum of shear wave, propagating in the material, at that second harmonic affects first only if there is a first harmonic's signal. The distance, at which the harmonic's amplitudes become equal, is obtained. The qualitative character of process of energy transfer is illustrated. The character of influence of media constants (density, shear modulus, limit intensity of shear strains) and wave parameters (frequency, wave number, first harmonic's initial amplitude) on a distance, corresponding to a state of equality of amplitudes of harmonics, is established.
\end{abstract}

Keywords: elasto-plastic media, second harmonic generation, nonlinear waves, energy transfer.

\section{Генерация второй гармоники сдвиговой волны в упруго-пластической среде}

\author{
Доронин А.М. ${ }^{\dagger}$, Ерофеев В.И. \\ †alexeydoronin@list.ru
}

Институт проблем машиностроения РАН, ул. Белинского, д. 85, 603024, г. Нижний Новгород, Россия

\begin{abstract}
Рассматривается упруго-пластическая среда, поведение которой описывается перекрестными зависимостями между первыми инвариантами тензоров и вторыми инвариантами девиаторов напряжений и деформаций, при этом зависимость от сдвиговых деформаций имеет квадратичный характер. Цель работы - определение возможности генерации второй гармоники сдвиговой волны, появление которой не описывается классической теорией упругости, и характера процесса перекачки энергии из первой гармоники во вторую. Для случая преобладания сдвиговых деформаций в предположении, что в среде существует только сдвиговая волна, проводится поиск решения нелинейного уравнения, определяющего динамическое поведение среды, в виде бегущих квазигармонических волн с медленно меняющимися амплитудами. В ходе рассмотрения системы укороченных уравнений для комплексных амплитуд, зависящих от времени и пройденного расстояния, определено, что взаимодействие гармоник носит несимметричный характер. При рассмотрении стационарного случая показано, что наличие даже малых пластических деформаций приведет к появлению удвоенной частоты (второй гармоники) в спектре сдвиговой волны, распространяющейся в материале, при этом вторая гармоника воздействует на первую лишь при наличии сигнала первой гармоники. Определено расстояние, на котором амплитуды первой и второй гармоник принимают равные значения. Проиллюстрирован качественный характер процесса перекачки энергии из первой гармоники во вторую. Определен характер влияния констант среды (плотность, модуль сдвига, предельная интенсивность сдвиговых деформаций) и параметров волны (частота, волновое число, начальная амплитуда первой гармоники) на расстояние, соответствующее равенству амплитуд первой и второй гармоник.
\end{abstract}

Ключевые слова: упруго-пластическая среда, генерация второй гармоники, нелинейные волны, перекачка энергии. 


\section{1. Введение}

В рамках нелинейной изотропной теории упругости невозможно описать генерацию второй гармоники при распространении сдвиговой волны в материале. Этот результат физически довольно очевиден, так как при распространении поперечных волн не изменяется плотность среды, и в изотропном твердом теле упругие напряжения при сдвиговых деформациях не зависят от знака деформации. Последнее, в частности, проявляется в том, что для плоских волн внутренняя энергия является четной функцией сдвиговых компонент тензора деформаций. Однако экспериментально вторая сдвиговая гармоника, «запрещенная» теорией упругости, наблюдалась при испытаниях образцов различных конструкционных материалов [1-3]. Одни авторы связывают появление этой гармоники с наличием остаточных внутренних напряжений [4], другие - с влиянием дислокаций $[5,6]$.

Покажем, что возникновение второй гармоники сдвиговой волны допускается в модели среды с малыми упруго-пластическими деформациями.

\section{2. Постановка задачи}

Уравнения, определяющие динамическое поведение упруго-пластической среды, получены в работе [7]. Для плоских продольно-поперечных волн система этих уравнений имеет вид:

$$
\begin{gathered}
{\left[\frac{K_{0}}{3}+\frac{4}{3} G_{0}\left(1-\frac{\sqrt{3}}{6} \frac{\sqrt{4(\partial u / \partial x)^{2}+3(\partial v / \partial x)^{2}}}{\Gamma_{S}}\right)-\right.} \\
\left.-\frac{8}{9} \frac{G_{0}}{\Gamma_{S}} \frac{\sqrt{3}(\partial u / \partial x)^{2}}{\sqrt{4(\partial u / \partial x)^{2}+3(\partial v / \partial x)^{2}}}\right] \cdot \frac{\partial^{2} u}{\partial x^{2}}- \\
-\frac{2 G_{0}}{3 \Gamma_{S}} \frac{\sqrt{3}(\partial u / \partial x)(\partial v / \partial x)\left(\partial^{2} v / \partial x^{2}\right)}{\sqrt{4(\partial u / \partial x)^{2}+3(\partial v / \partial x)^{2}}}=\rho \frac{\partial^{2} u}{\partial t^{2}} \\
{\left[\begin{array}{c}
G_{0}\left(1-\frac{1}{2} \frac{\sqrt{3}}{3} \frac{\sqrt{4(\partial u / \partial x)^{2}+3(\partial v / \partial x)^{2}}}{\Gamma_{S}}\right)- \\
\left.-\frac{\sqrt{3}}{2} \frac{G_{0}}{\Gamma_{S}} \frac{(\partial v / \partial x)^{2}}{\sqrt{4(\partial u / \partial x)^{2}+3(\partial v / \partial x)^{2}}}\right] \cdot \frac{\partial^{2} v}{\partial x^{2}}- \\
-\frac{2 G_{0}}{3 \Gamma_{S}} \frac{\sqrt{3}(\partial v / \partial x)(\partial u / \partial x)\left(\partial^{2} u / \partial x^{2}\right)}{\sqrt{4(\partial u / \partial x)^{2}+3(\partial v / \partial x)^{2}}}=\rho \frac{\partial^{2} v}{\partial t^{2}}
\end{array}\right.}
\end{gathered}
$$

Здесь $u, v-$ перемещения по продольной и поперечной оси соответственно, $G_{0}-$ модуль сдвига, $K_{0}-$ модуль объемного расширения, $\Gamma_{S}$ - предельная интенсивность деформаций сдвига, Г- интенсивность сдвиговых деформаций, $0 \leq \Gamma / \Gamma_{s} \leq 1, \rho-$ плотность, $t-$ время, $x-$ координата. В предельном случае (при $\Gamma_{s}=\infty$ ) слагаемые, определяемые пластическими свойствами материала, исчезнут, и система (1) будет соответствовать линейноупругой задаче.

\section{3. Поиск решений}

В случае, когда преобладают сдвиговые деформации, т.е. $\partial v / \partial x \gg \partial u / \partial x$

$$
\Gamma=\sqrt{4(\partial u / \partial x)^{2}+3(\partial v / \partial x)^{2}} \approx \sqrt{3}(\partial v / \partial x) .
$$

Система уравнений (1) примет вид

$$
\left\{\begin{array}{l}
\left(\frac{K_{0}}{3}+\frac{4}{3} G_{0}\right) u_{x x}-\frac{2}{3} \frac{G_{0}\left(v_{x} u_{x x}+u_{x} v_{x x}\right)}{\Gamma_{S}}=\rho u_{t t}, \\
G_{0} v_{x x}-\frac{G_{0}}{\Gamma_{S}}\left(v_{x} v_{x x}+2 / 3 u_{x} u_{x x}\right)=\rho v_{t t} .
\end{array}\right.
$$

Если предположить, что имеется лишь сдвиговая волна $(u \equiv 0)$, то останется только второе уравнение:

$$
G_{0} v_{x x}-\frac{G_{0}}{\Gamma_{S}} v_{x} v_{x x}=\rho v_{t t}
$$

Если считать среду слабо нелинейной, то амплитуды волн будут изменяться на малую величину при прохождении волной расстояния порядка длины волны, то есть амплитуды волн будут медленно меняющимися функциями координат и времени. В соответствии с этим решение уравнения (2) будем искать в виде квазигармонических волн [8]:

$$
\begin{aligned}
& v=A_{1}(\varepsilon x, \varepsilon t) e^{i(\omega t-k x)}+A_{2}(\varepsilon x, \varepsilon t) e^{2 i(\omega t-k x)}+ \\
& +\bar{A}_{1}(\varepsilon x, \varepsilon t) e^{-i(\omega t-k x)}+\bar{A}_{2}(\varepsilon x, \varepsilon t) e^{-2 i(\omega t-k x)} .
\end{aligned}
$$

Здесь $A_{1}, A_{2}$ - комплексные амплитуды, $\omega-$ частота, $k-$ волновое число, $\varepsilon-$ малый параметр. Подставляя выражения (3) в уравнение (2), получаем в первом приближении по $\varepsilon$ следующую систему укороченных уравнений для комплексных амплитуд, зависящих от времени и пройденного расстояния:

$$
\left\{\begin{array}{l}
A_{1 t}+V_{\phi} A_{1 x}-\frac{G_{0}}{\rho \Gamma_{S} \varepsilon \omega} \bar{A}_{1} A_{2} k^{3}=0, \\
A_{2 t}+V_{\phi} A_{2 x}-\frac{G_{0}}{4 \rho \Gamma_{S} \varepsilon \omega} A_{1}^{2} k^{3}=0 .
\end{array}\right.
$$

Здесь $V_{\phi}=\omega / k-$ фазовая скорость. Из системы видно, что взаимодействие первой и второй гармоник имеет несимметричный характер: квадрат амплитуды первой гармоники входит в уравнение второй гармоники в виде вынуждающей силы, а амплитуда второй гармоники входит в уравнение для первой параметрическим образом. Следовательно, первая гармоника всегда генерирует вторую гармонику, вторая же гармоника воздействует на первую лишь при наличии сигнала первой гармоники.

В стационарном случае $(\partial / \partial t=0)$

$$
\left\{\begin{array}{l}
A_{1 x}-4 M \bar{A}_{1} A_{2}=0, \\
A_{2 x}+M A_{1}^{2}=0,
\end{array}\right.
$$

где

$$
M=G_{0} k^{4} / 4 \rho \Gamma_{S} \varepsilon \omega^{2} .
$$

В уравнениях (4) перейдем к действительным амплитудам и фазам. Для этого положим

$$
\begin{aligned}
& A_{1}(x)=B_{1}(x) e^{i S_{1}(x)} \\
& A_{2}(x)=B_{2}(x) e^{i S_{2}(x)}
\end{aligned}
$$

Система (4) примет вид

$$
\left\{\begin{array}{l}
d B_{1} / d x-4 M B_{1} B_{2} \cos \Delta=0, \\
d B_{2} / d x+M B_{1}^{2} \cos \Delta=0, \\
d \Delta / d x+M\left(8 B_{2}-B_{1}^{2} / B_{2}\right) \sin \Delta=0,
\end{array}\right.
$$


где $\Delta=2 S_{1}-S_{2}$. Из первых двух уравнений системы (5) можно получить интеграл энергии

$$
B_{1}^{2}(x)+4 B_{2}^{2}(x)=\text { const } .
$$

Для получения решения удобно подставить первые два уравнения системы в третье:

$$
\frac{d \Delta}{d x}=-\operatorname{tg} \Delta \frac{d}{d x}\left(\ln B_{1}^{2} B_{2}\right) .
$$

Уравнение (6) легко интегрируется, что позволяет получить следующее выражение:

$$
\sin \Delta B_{1}^{2} B_{2}=\sin \Delta(0) B_{1}^{2}(0) B_{2}(0) .
$$

Если положить $B_{2}(0)=0$, то $\sin \Delta=0, \Delta=\pi k$. Для реализации случая перекачки энергии из первой гармоники во вторую выберем $\Delta=\pi$. Тогда

$$
\left\{\begin{array}{l}
d B_{1} / d x=-4 M B_{1} B_{2}, \\
d B_{2} / d x=M B_{1}^{2} .
\end{array}\right.
$$

При наличии интеграла энергии можно записать

Отсюда имеем

$$
d B_{2} / d x=M\left(B_{1}^{2}(0)-4 B_{2}^{2}\right) .
$$

$$
\begin{aligned}
& B_{1}(x)=B_{1}(0) \operatorname{ch}^{-1}\left(2 B_{1}(0) M x\right) \\
& B_{2}(x)=B_{1}(0) / 2 \operatorname{th}\left(2 B_{1}(0) M x\right)
\end{aligned}
$$

Решение (7) представлено на рисунке 1.

Как видно, с ростом $x$ происходит перекачка энергии во вторую гармонику. Амплитуды гармоник принимают равные значения при $2 B_{1}(0) M x \approx 1.44$.

Определим расстояние $x^{*}$, соответствующее равенству амплитуд первой и второй гармоник:

$$
x^{*} \approx \frac{0.77}{B_{1}(0) M}=\frac{2.88 \Gamma_{S} \varepsilon \omega^{2}}{B_{1}(0) k^{4} c_{\tau}^{2}},
$$

где $c_{\tau}=\sqrt{G_{0} / \rho}-$ скорость сдвиговой упругой волны.

Видно, что данное расстояние обратно пропорционально сдвиговому модулю упругости и величине начальной амплитуды, а также прямо пропорционально квадрату частоты волны (т.е., из более высокочастотной волны энергия перекачивается медленнее).

Благодарность/Aknowledgements. Работа выполнялась при финансовой поддержке Российского научного бонда (Грант № 14-19-01637).

\section{Литература/References}

1. Zarembo L.K., Shklovskaya-Kordy V.V. SSP.12, 3637 (1970) (in Russian) [Л. К. Зарембо, В.В. ШкловскаяКорди. ФТТ. 12, 3637 (1970)].

2. Ermilin K.K., Zarembo L.K., Krassil'nikov V.A., Mezintsev E.D., Prokhorov V.M., Hilkov K.B. Phys. Met. Metallogr.36, 640 (1973) (in Russian) [К.К. Ермилин,

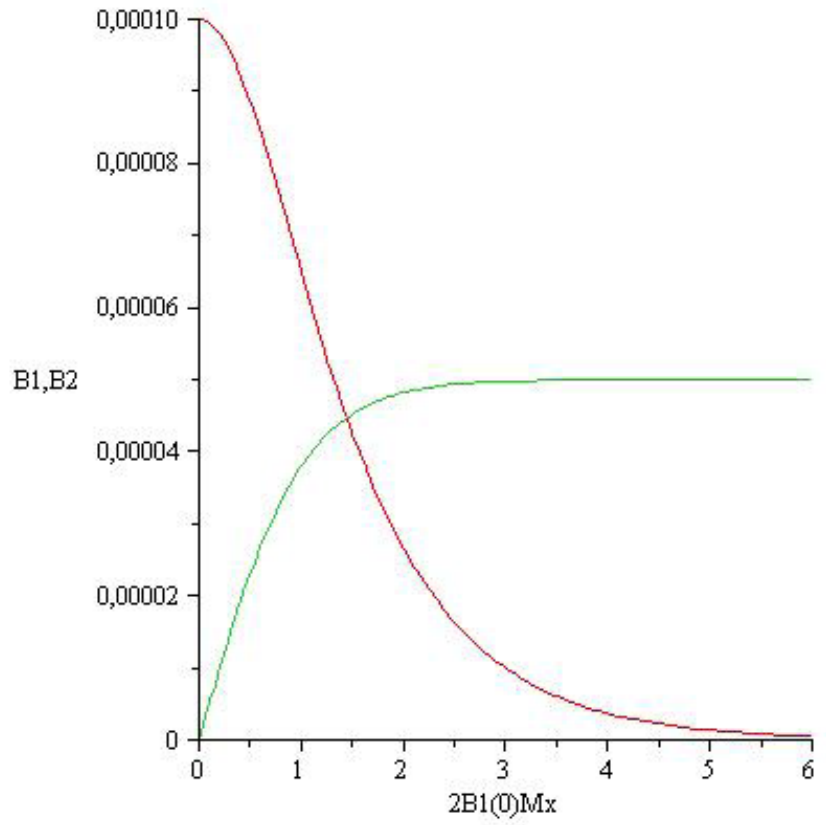

Рис. 1. Процесс перекачки энергии во вторую гармонику в соответствии с решением (8) (начальная амплитуда первой гармоники $0.0001 \mathrm{~m})$.

Fig. 1. The process of energy transfer to second harmonic with respect to solution (8) (initial amplitude of second harmonic equals to $0.0001 \mathrm{~m})$.

Л.К. Зарембо, В.А. Красильников, Е.Д. Мезинцев, В.М. Прохоров, К.Б. Хилков. ФММ. 36, 640 (1973)].

3. Erofeev V.I., Mishakin V.V., Rodyushkin V.M., Sharabanova A.V. Defektoskopiya. (4), 28 (2006) (in Russian) [В.И. Ерофеев, В.В. Мишакин, В.М. Родюшкин, А.В. Шарабанова. Дефектоскопия. (4), 28 (2006)].

4. Zarembo L.K., Prokhorov V.M. Acoust. Journal. 21,198 (1975) (in Russian) [Л.К. Зарембо, В.М. Прохоров. Акуст.журн. 21, 198 (1975)].

5. Mirsaev I.F, Nikolaev V.V., Taluts G.G. Phys. Met. Metallogr. 31, 1128 (1971) (in Russian) [И.Ф. Мирсаев, В.В. Николаев, Г.Г. Талуц. ФММ. 31, 1128 (1971)].

6. Lyamov V.E. SSP. 23, 1483 (1981) (in Russian) [B.E. Лямов. ФТТ. 23, 1483 (1981)].

7. Bakushev S.V. Problems of strength and plasticity. 76, 114 (2014) (in Russian) [C.В. Бакушев. Проблемы прочности и пластичности. 76, 114 (2014)].

8. Rudenko O.V., Soluian S.I. Theoretical foundations of nonlinear acoustics. Moscow, Science (1975), 287 (in Russian) [О.В. Руденко, С.И. Солуян. Теоретические основы нелинейной акустики, М., Наука (1975) 287]. 